\title{
Identification of miRNA biomarkers of pneumonia using RNA-sequencing and bioinformatics analysis
}

\author{
SAI HUANG ${ }^{1 *}$, CONG FENG $^{2 *}$, YONG-ZHI ZHAI ${ }^{2 *}$, XUAN ZHOU $^{2}$, \\ BEI LI ${ }^{2}$, LI-LI WANG ${ }^{2}$, WEI CHEN ${ }^{2}$, FA-QIN LV ${ }^{3}$ and TAN-SHI LI ${ }^{2}$ \\ Departments of ${ }^{1}$ Hematology, ${ }^{2}$ Emergency and ${ }^{3}$ Ultrasound, \\ People's Liberation Army General Hospital, Beijing 100853, P.R. China
}

Received July 15, 2016; Accepted August 10, 2016

DOI: $10.3892 /$ etm.2017.4151

\begin{abstract}
Pneumonia is a lower respiratory tract infection that causes dramatic mortality worldwide. The present study aimed to investigate the pathogenesis of pneumonia and identify microRNA (miRNA) biomarkers as candidates for targeted therapy. RNA from the peripheral blood plasma of participants with pneumonia (severe, $n=9$; non-severe, $n=9$ ) and controls $(n=9)$ was isolated and paired-end sequencing was performed on an Illumina HiSeq4000 system. Following the processing of raw reads, the sequences were aligned against the Genome Reference Consortium human genome assembly 38 reference genome using Bowtie 2 software. Reads per kilobase of transcript per million mapped read values were obtained and the limma software package was used to identify differentially expressed miRNAs (DE-miRs). Then, DE-miR targets were predicted and subjected to enrichment analysis. In addition, a protein-protein interaction (PPI) network of the predicted targets was constructed. This analysis identified 11 key DE-miRs in pneumonia samples, including 6 upregulated miRNAs (including hsa-miR-34a and hsa-miR-455) and 5 downregulated miRNAs (including hsa-let-7f-1). All DE-miRs kept their upregulation/downregulation pattern in the control, non-severe pneumonia and severe pneumonia samples. Predicted target genes of DE-miRs in the subjects with non-severe pneumonia vs. the control and the subjects with severe pneumonia vs. the non-severe pneumonia group were markedly enriched in the adherens
\end{abstract}

Correspondence to: Dr Tan-Shi Li, Department of Emergency, People's Liberation Army General Hospital, 28 Fuxing Road, Beijing 100853, P.R. China

E-mail: 1ts301@sohu.com

Dr Fa-Qin Lv, Department of Ultrasound, People's Liberation Army General Hospital, 28 Fuxing Road, Beijing 100853, P.R. China E-mail:1vjin8912@163.com

*Contributed equally

Key words: pneumonia, RNA-sequencing, miRNA, target, protein-protein interaction, notch signaling pathway junction and Wnt signaling pathways. $K A L R N$, Ras homolog family member A (RHOA), $\beta$-catenin (CTNNB1), RNA polymerase II subunit $\mathrm{K}(P O L R 2 K)$ and amyloid precursor protein $(A P P)$ were determined to encode crucial proteins in the PPI network constructed. KALRN was predicted to be a target of hsa-mir-200b, while RHOA, CTNNB1, POLR $2 \mathrm{~K}$ and $A P P$ were predicted targets of hsa-let-7f-1. The results of the present study demonstrated that hsa-let-7f-1 may serve a role in the development of cancer and the Notch signaling pathway. Conversely, hsa-miR-455 may be an inhibitor of pneumonia pathogenesis. Furthermore, hsa-miR-200b might promote pneumonia via targeting KALRN.

\section{Introduction}

Lower respiratory tract infections (LRTIs), including pneumonia, are a primary cause of infection-based mortalities. The World Health Organization estimates, that $~ 3,500,000$ mortalities are caused by this infection annually (1). Community-acquired pneumonia (CAP) is a severe type of pneumonia that typically requires hospitalization. CAP is a primary factor for adult mortality, and for those who survive, mortality in the following years remains high $(2,3)$. Among adolescents, it is estimated that pneumonia accounts for $15 \%$ of mortalities worldwide (4). Although the occurrence of childhood pneumonia has been reduced, the decline has been small. Reportedly, $\sim 950,000$ individuals $<5$ years old succumbed to pneumonia in 2013 (5).

Severe pneumonia requires treatment in an intensive care unit. Viral infection is a primary factor for the mortality of patients with severe pneumonia (6) and a number of viruses, such as the respiratory syncytial virus, have been identified in infants and children with severe pneumonia (7). Although vaccines against viruses, including Haemophilus influenzae type b and Streptococcus pneumoniae, have been introduced in numerous locations, achieving sufficient coverage in developed countries remains a challenge (5). Therefore, it is important to develop novel and improved methods of diagnosing and treating patients with pneumonia.

Targeted therapy has been widely used for the management of numerous types of cancer and diseases. A previous study reported that the plasminogen activator inhibitor-1 gene $4 \mathrm{G} / 5 \mathrm{G}$ polymorphism may be used as a marker for the diagnosis of 
severe pneumonia (8). Elevated levels of procalcitonin and C-reactive protein are positively correlated with the severity of CAP (9). MicroRNAs (miRNAs or miRs) are non-coding RNAs of $\leq 200$ base pairs in length that can regulate gene expression at transcriptional or post-transcriptional levels, influencing cellular activities and disease progression (10). Few miRNAs have been detected in the pathogenesis of pneumonia. Abd-El-Fattah et al (11) demonstrated that miR-155, miR-21 and miR-197 were markedly increased in patients with lung cancer or pneumonia. Another previous study, using miRNA microarray data from patients with severe pneumonia infected with the influenza A subtype H1N1, identified a number of differentially expressed miRNAs (DE-miRs; upregulated, hsa-miR-374a, hsa-miR-875-5p, hsa-miR-342-3p, hsa-miR-150 and hsa-miR-15b; downregulated, hsa-miR-29c, hsa-miR-1247 and hsa-miR-1233) in patients with severe pneumonia, compared with patients with non-severe pneumonia or healthy controls. In addition, upregulated miRNAs that associated with severe pneumonia primarily affect transforming growth factor-, apoptosis and Wnt/ $\beta$-catenin signaling pathways (12). Despite these discoveries, the targets of these DE-miRs, and their target's function and potential interactions, remain unknown.

RNA-sequencing (RNA-seq) has been successfully used to detect gene alterations, gene-fusions and somatic mutations, and is a markedly more precise method for the detection of the transcripts compared with other methods $(13,14)$. The present study utilized RNA-seq to detect miRNA expression in patients with severe pneumonia, patients with non-severe pneumonia and healthy controls. The results of these groups were compared to identify DE-miRs. In addition, target genes of these DE-miRs were predicted and put through enrichment analysis. Furthermore, a protein-protein interaction (PPI) network of the predicted targets was constructed to investigate the potential regulatory relationships of them at the protein level. Through these comprehensive bioinformatics analyses, the present study aimed to explore the pathogenesis of pneumonia and identify important miRNA therapeutic targets.

\section{Materials and methods}

Sample collection. Three types of samples were collected in the present study: Patients with severe pneumonia (severe sample: $n=9$; mean age, $65.2 \pm 11.9$ years; all male), non-severe pneumonia (non-severe sample: $n=9$; mean age, 21.3 \pm 2.7 years; all male) and healthy individuals (controls: $n=9$; mean age, $19.6 \pm 2.2$ years; all male). The patients were hospitalized at the People's Liberation Army General Hospital (PLA; Beijing, China) between June and December 2013. The average duration of stay in the hospital was 18.2 days in the severe group and 14.6 days in the non-severe group. Severe pneumonia was diagnosed if one or more of the following conditions, based on the previously described CAP guidelines (15), was present: i) Disturbance of consciousness; ii) respiratory rate $\geq 30$ breaths/min; iii) $\mathrm{PaO}_{2}<60 \mathrm{mmHg}, \mathrm{PaO}_{2} / \mathrm{FiO}_{2}$ $<300 \mathrm{mmHg}$, with mechanical ventilation required; vi) systolic arterial pressure $<90 \mathrm{mmHg}$; v) complications related to septic shock; vi) chest X-ray showing bilateral lung involvement or lung lesion expansion of $\geq 50 \%$ within $48 \mathrm{~h}$ following hospitalization; and vii) oliguria, urine volume $<20$ or $>80 \mathrm{ml} / \mathrm{h}$, or acute renal failure requiring dialysis. The present study was approved by the Medical Ethics Committee of the PLA General Hospital and all participants gave informed consent.

RNA isolation and sequencing. Total RNA in the peripheral blood plasma was isolated using the mirVan miRNA Isolation kit (Ambion; Thermo Fisher Scientific, Inc., Waltham, MA, USA) and purified using the miRNeasy Mini kit (Qiagen, $\mathrm{GmbH}$, Hilden, Germany), according to the manufacturer's instructions. Then, the isolated RNA was quantified using a spectrophotometer (NanoDrop, Thermo Fisher Scientific, Inc., Wilmington, DE, USA). Purified RNA was frozen with dry ice and stored at $-80^{\circ} \mathrm{C}$ until required.

As the quantity of RNA extracted was relatively low, the nine patients within each group (severe pneumonia, non-severe pneumonia and control) were randomly divided into three sub-groups. Within each subgroup, the three patients' RNAs were mixed equally and used as one sample for sequencing. Therefore, there were three samples from each group [severe pneumonia (WLL1, WLL2 and WLL3), non-severe pneumonia (WLL4, WLL5 and WLL6) and the control (WLL7, WLL8 and WLL9)].

Following extraction and purification, an RNA library was constructed using the NEBNext Ultra RNA Library Prep kit for Illumina (New England Biolabs, Inc., Ipswich, MA, USA) based on the manufacturer's instructions. In brief, four steps were performed: i) Total RNA $(1 \mu \mathrm{g})$ was sheared into fragments (200-500 nucleotides) in the NEBNext First Strand Synthesis Reaction Buffer and the fragments were utilized to generate the double-stranded cDNA; ii) the cDNA was end-repaired and ligated with Illumina-specific adaptors; iii) size selection of the library was performed using $200 \mathrm{bp}$ inserts to select suitable fragments for polymerase chain reaction (PCR) amplification; iv) PCR was performed using Phusion High-Fidelity DNA polymerase (New England Biolabs, cat. no. F-530S) and the products were purified with a QIAquick Nucleotide Removal kit (Qiagen, no. 28304). The constructed RNA library was sequenced on an Illumina HiSeq 4000 system using 2x50 base pairs paired-end sequencing.

Processing of the sequencing data. FASTX-Toolkit software (ver. 0.0.13, http://hannonlab.cshl.edu/fastx_toolkit) was used to remove the $3^{\prime}$ adaptor primer from the raw read. In addition, bases with a quality score $<20$ were eliminated. If $30 \%$ of bases in a sequence were eliminated, the entire sequence was discarded and did not undergo further analysis.

Identification of DE-miRs. Following processing, the remaining reads were aligned to the Genome Reference Consortium human genome assembly 38 (GRCH38) reference genome using Bowtie2 software (version 2.1.0, www.sourceforge.net/projects/bowtie-bio/files/bowtie2). In the alignment, each read was allowed $\leqq 8$ base mismatches and only reads with a quality score $>60$ were retained. In addition, considering RNA splicing, the length of reads was not taken into account as a restriction when performing the alignment.

The latest (June 2016) human miRNA sequences and their locations were downloaded from the miRbase database (16) and the aligned sequences of each miRNA counted using perl programing, written by the present authors. The total quantity of 


\section{Non-severe vs control Severe vs non-severe}

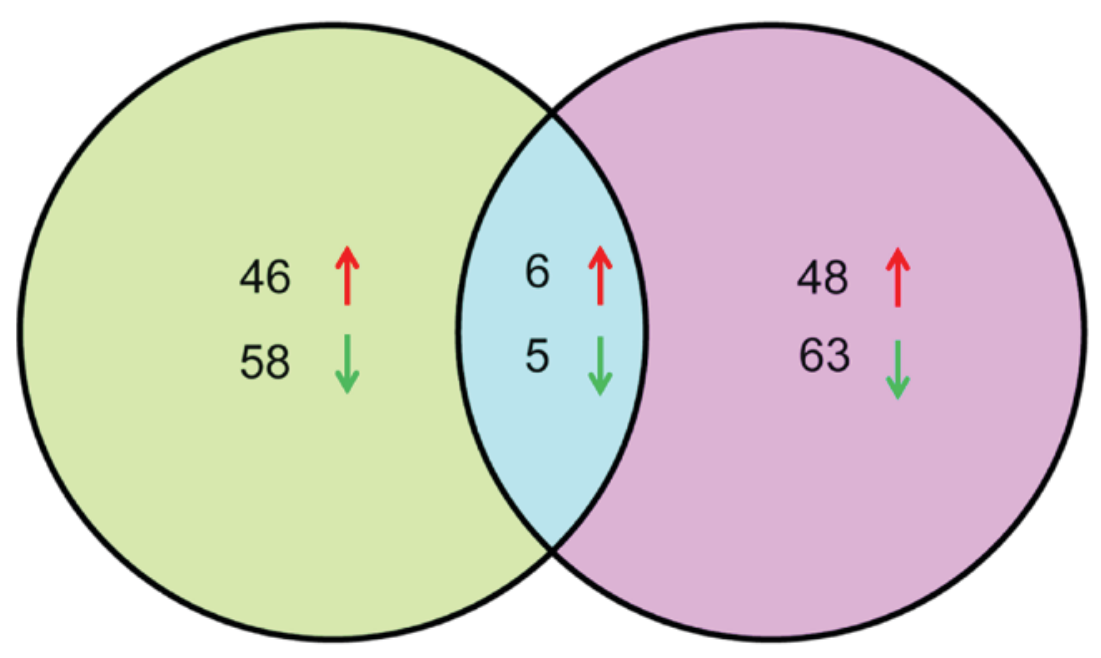

Figure 1. Venn analysis of DE-miRs from the comparison of the non-severe pneumonia and control groups, and the severe and non-severe pneumonia groups. Upward arrows in red represent upregulation and downward arrows in blue represent downregulation. DE-miRs, differentially-expressed microRNAs.

each miRNA in each sample was used to calculate the number of mapped reads per kilobase per million reads (RPKM), which were normalized using DESeq software (version 1.24.0; www. bioconductor.org/packages/release/bioc/html/DESeq.html). Then, DE-miRs were selected based on un-paired $t$-tests in limma (version 3.22.7; www.bioconductor.org/packages/3.0/ bioc/html/limma.html) (17), followed by adjustment using the Benjamini-Hochberg method (18). The adjusted P-value was deemed to be the false discovery rate (FDR). The cut-off for DE-miRs between different types of samples (non-severe vs. control; severe vs. non-severe) was a log 2 fold-change (FC) $>1$ and FDR $<0.5$. Afterwards, a Venn diagram was delineated to identify the overlapped DE-miRs in the two comparisons and their expression trends in the three different types of samples were portrayed. Two-way hierarchical cluster analysis was performed to present the expression of DE-miRs in the three types of samples.

DE-miR target prediction. Gene sequences of GRCH38 reference genome corresponding to the DE-miRs were downloaded. miRanda software (ver. 1.0, www.microrna.org) (19) was used to predict target genes of these DE-miRs. Efficiency of the microRNA target sites predicted by this software was scored and ranked by the mirSVR algorithm. The mirSVR score reflects the empirical probability that a gene is downregulated by a specific microRNA (20). As each DE-miR had multiple targets, only genes with a mirSVR score $<-1.2$ were considered to be targets.

Enrichment analysis of the predicted targets. Gene ontology (GO; www. geneontology.org) (21) and Kyoto Encyclopedia of Genes and Genomes (KEGG; www. genome.jp/kegg/pathway.html) (22) enrichment analyses were performed using the Database for Annotation, Visualization and Integration Discovery (DAVID; version 6.7; https://david.ncifcrf.gov) (23). Pathway enrichment analysis of targets was conducted using clusterProfiler software (ver. 2.8, www.bioconductor.org/packages/2.8/bioc/html/
clusterProfiler.html) (24). Function and pathways of the reference species were deposited in the GO and KEGG databases. Fisher's exact test was used for statistical analysis and the threshold for significant function and pathway categories of the target genes was $\mathrm{P}<0.05$, compared with those of the reference species.

Construction of PPI networks. To identify potential regulatory relationships of targets of the DE-miRs at protein level, the Search Tool for the Retrieval of Interacting Genes (STRING; ver. 10.0; www.string-db.org) database (25) was used to build PPI networks of the targets. DEGs with a combined score $>0.9$ were entered into the STRING database. PPI networks were visualized using the Cytoscape software (ver. 3.1.0; www.cytoscape.org) (26). A node in the network is defined as an encoded protein of a target gene and the nodal degree represents the linked gene numbers of this node. In addition, a literature search was conducted to identify reports of interactions between these targets. The key search terms used were the name of the specific target gene and the gene that linked to it. These were: 'CTNNB1' AND 'CRBE', or 'CTNNB1' AND 'FOXO1', OR 'CTNNB1' AND 'XPO1'.

\section{Results}

Identification of DE-miRs. Under the inclusion criteria of $\log 2 \mathrm{FC}>1$ and FDR $<0.05$, a total of $237 \mathrm{DE}-\mathrm{miRs}$ were identified. From the comparison of the non-severe and control groups, there were 52 upregulated and 63 downregulated miRNAs, whereas following the comparison of the severe and non-severe groups, there were 54 upregulated and 68 downregulated miRNAs (Fig. 1). Venn analysis identified 11 overlapping DE-miRs from both comparisons, of which 6 were upregulated (hsa-miR-200b, hsa-miR-483, hsa-miR-34a, hsa-miR-193b, hsa-miR-455 and hsa-miR-95), and 5 were downregulated (hsa-miR-3617, hsa-miR-664b, hsa-miR-4485, hsa-miR-3161 and hsa-let-7f-1; Fig. 1). Based on this result, the levels of expression of the $11 \mathrm{DE}-\mathrm{miRs}$ in the three types of 
A

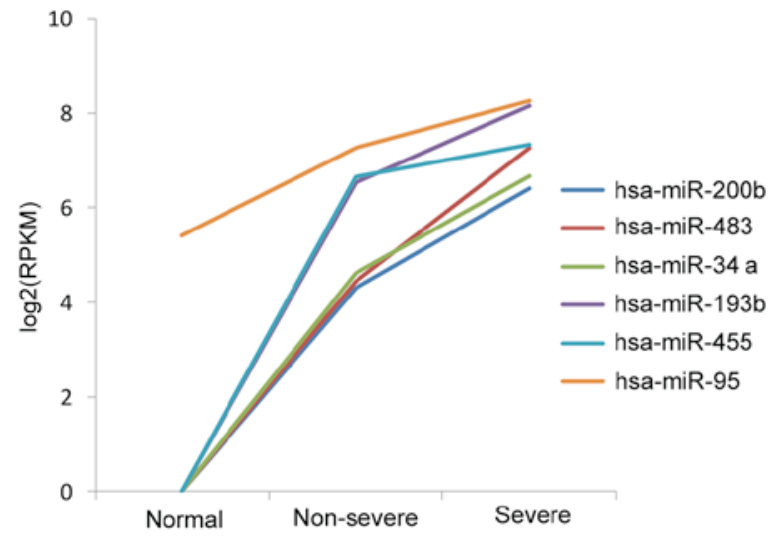

B

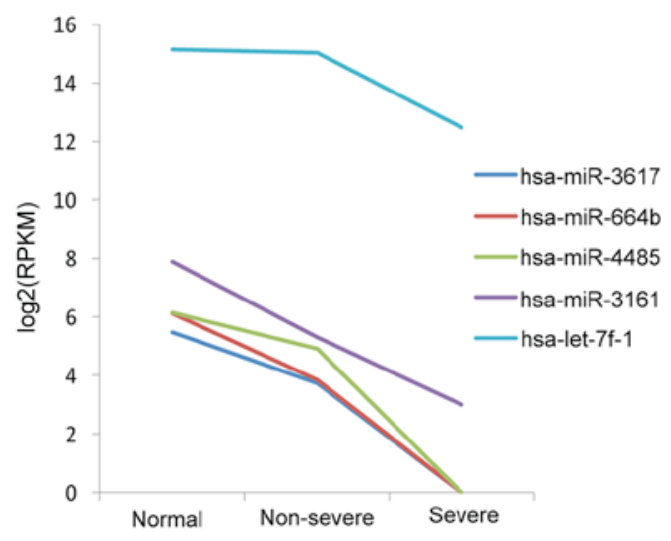

Figure 2. Expression of 11 key DE-miRs in the plasma of the peripheral blood of the severe pneumonia, non-severe pneumonia and control groups. Key DE-miRs that are (A) upregulated and (B) downregulated. DE-miRs, differentially expressed microRNAs. RPKM, per kilobase per million reads.

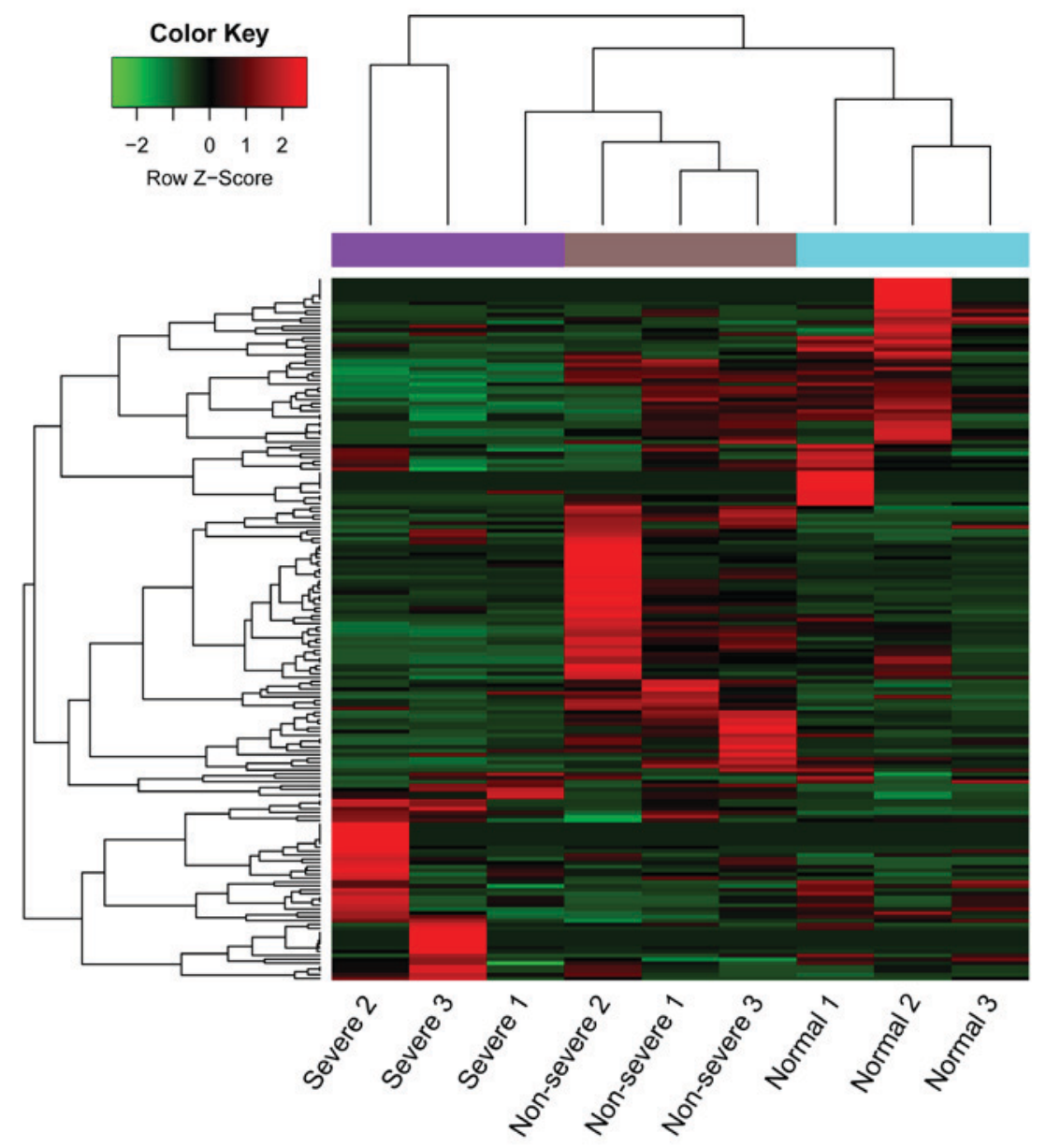

Figure 3. Heatmap from clustering analysis of DE-miRs in the plasma of the peripheral blood of the severe pneumonia, non-severe pneumonia and control groups. The $\mathrm{x}$-axis denotes samples and the $\mathrm{y}$-axis denotes miRNA. The $\mathrm{Z}$-score indicates the parameter used for the calculation of miRNA expression based on raw reads. A value of $>0$ was deemed to indicate upregulation (red) and a value $<0$ was deemed to indicate downregulation (green). The purple, brown and blue panels at the top of the heat map indicate severe, non-severe and control samples, respectively. DE-miRs, differentially expressed microRNAs.

samples were assessed. It was observed that the 6 miRNAs were upregulated and 5 were downregulated. These miRNAs were up and downregulated, respectively, in the pneumonia groups compared with the controls, which may reflect the three phases of pneumonia progression (Fig. 2). A heat map of the cluster analysis is presented in Fig. 3, which indicated that the three types of samples could be identified by their differing expression of DE-miRs. 
Table I. Top 10 enriched functions of the target genes of DE-miRs from the comparison of the non-severe pneumonia and control groups.

\begin{tabular}{|c|c|c|c|}
\hline GO ID no. & Function & Count & P-value \\
\hline GO:0009653 & Anatomical structure morphogenesis & 757 & $<1.0 \mathrm{E}^{-16}$ \\
\hline GO:0009893 & Positive regulation of metabolic process & 938 & $<1.0 \mathrm{E}^{-16}$ \\
\hline GO:0010604 & Positive regulation of macromolecule metabolic process & 770 & $<1.0 \mathrm{E}^{-16}$ \\
\hline GO:0019222 & Regulation of metabolic process & 1,637 & $<1.0 \mathrm{E}^{-16}$ \\
\hline GO:0032502 & Developmental process & 1,439 & $<1.0 \mathrm{E}^{-16}$ \\
\hline GO:0044767 & Single-organism developmental process & 1,424 & $<1.0 \mathrm{E}^{-16}$ \\
\hline GO:0048518 & Positive regulation of biological process & 1,355 & $<1.0 \mathrm{E}^{-16}$ \\
\hline GO:0048522 & Positive regulation of cellular process & 1,188 & $<1.0 \mathrm{E}^{-16}$ \\
\hline GO:0007275 & Multicellular organismal development & 1,231 & $2.22 \mathrm{E}^{-16}$ \\
\hline GO:0045893 & Positive regulation of transcription, DNA-templated & 433 & $2.22 \mathrm{E}^{-16}$ \\
\hline
\end{tabular}

Count indicates number of the identified target genes that were enriched in a specific GO term. GO, gene ontology; DE-miRs, differentially expressed microRNAs.

Table II. Top 10 enriched functions of the target genes of DE-miRs from the comparison of the severe and non-severe pneumonia groups.

\begin{tabular}{llrr}
\hline GO ID no. & \multicolumn{1}{c}{ Function } & Count & P-value \\
\hline GO:0007275 & Multicellular organismal development & 1,252 & $<1.00 \mathrm{E}^{-16}$ \\
GO:0009653 & Anatomical structure morphogenesis & 777 & $<1.00 \mathrm{E}^{-16}$ \\
GO:0009893 & Positive regulation of metabolic process & 943 & $<1.00 \mathrm{E}^{-16}$ \\
GO:0019222 & Regulation of metabolic process & 1,646 & $<1.00 \mathrm{E}^{-16}$ \\
GO:0032502 & Developmental process & 1,447 & $<1.00 \mathrm{E}^{-16}$ \\
GO:0044767 & Single-organism developmental process & 1,433 & $<1.00 \mathrm{E}^{-16}$ \\
GO:0048518 & Positive regulation of biological process & 1,348 & $<1.00 \mathrm{E}^{-16}$ \\
GO:0048856 & Anatomical structure development & 1,300 & $<1.00 \mathrm{E}^{-16}$ \\
GO:0010604 & Positive regulation of macromolecule metabolic process & 768 & $1.11 \mathrm{E}^{-16}$ \\
GO:0016043 & Cellular component organization & 1,484 & $4.44 \mathrm{E}^{-16}$ \\
\hline
\end{tabular}

Count indicates the number of genes that were enriched in a specific GO term. GO, gene ontology; DE-miRs, differentially expressed microRNAs.

Enrichment analysis of the predicted targets of the DE-miRs. A total of 9,026 targets of the DE-miRs were identified (non-severe pneumonia vs. the control group, 4,498; severe pneumonia vs. non-severe pneumonia, 4,528). Targets of DE-miRs from the comparison of non-severe pneumonia and the control groups were primarily enriched in anatomical structure morphogenesis and metabolism regulation-associated functions (Table I), and pathways involved in cancer, ubiquitin mediated proteolysis, adherens junction and Wnt signaling (Fig. 4A). Target genes of DE-miRs from the comparison of the severe pneumonia and non-severe pneumonia groups produced similar results in the enrichment analysis (Table II; Fig. 4B).

Pathway enrichment of the 11 overlapping DE-miRs. Following the integration of KEGG pathway analysis of targets of the 11 DE-miRs and their miRNA-target interactions, the enriched pathways of 5 DE-miRs were obtained (Fig. 5). The miRNA hsa-let-7f-1 was significantly enriched in a number of pathways compared with the reference species in the GO and KEGG databases. These included 'pathways in cancer' $(\mathrm{P}=0.001)$, 'basal cell carcinoma' $(\mathrm{P}=0.002)$ and 'Notch signaling pathways' $(\mathrm{P}=0.003)$; hsa-miR-200b was enriched in 'SNARE interactions in vesicular transport' $(\mathrm{P}=0.009)$ and 'basal transcription factors' $(\mathrm{P}=0.010)$; hsa-miR-34a was enriched in 'RNA transport' $(\mathrm{P}=0.018)$, 'colorectal cancer' $(\mathrm{P}=0.022)$, 'leishmaniasis' $(\mathrm{P}=0.030)$, 'B cell receptor signaling pathway' $(\mathrm{P}=0.031)$ and 'riboflavin metabolism' $(\mathrm{P}=0.040)$; hsa-miR-455 was enriched in 'riboflavin metabolism' $(\mathrm{P}<0.001)$, 'ascorbate and aldarate metabolism' $(\mathrm{P}<0.001)$ and 'drug metabolism-other enzymes' $(\mathrm{P}<0.001)$; and hsa-miR-483 was enriched in pathways associated with 'long term depression' $(\mathrm{P}=0.006)$, the 'pentose phosphate pathway' $(\mathrm{P}=0.045)$ and 'linoleic acid metabolism' $(\mathrm{P}=0.050)$.

PPI network of 11 key targets of DE-miRs. A PPI network of targets of the DE-miRs was constructed (Fig. 6), as 
A

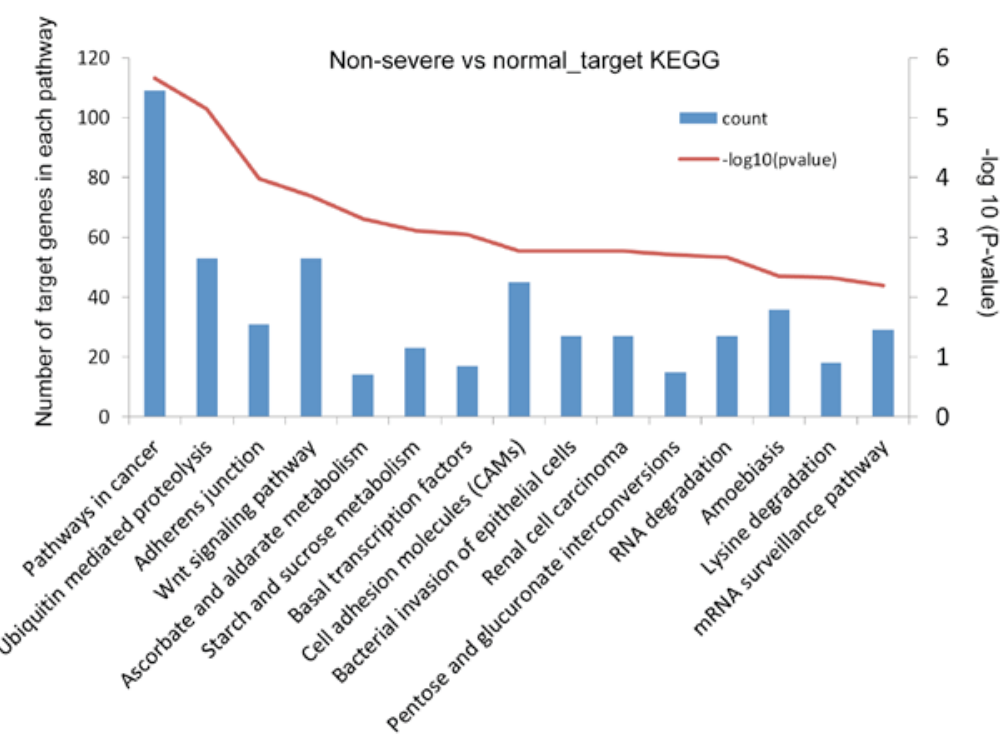

B

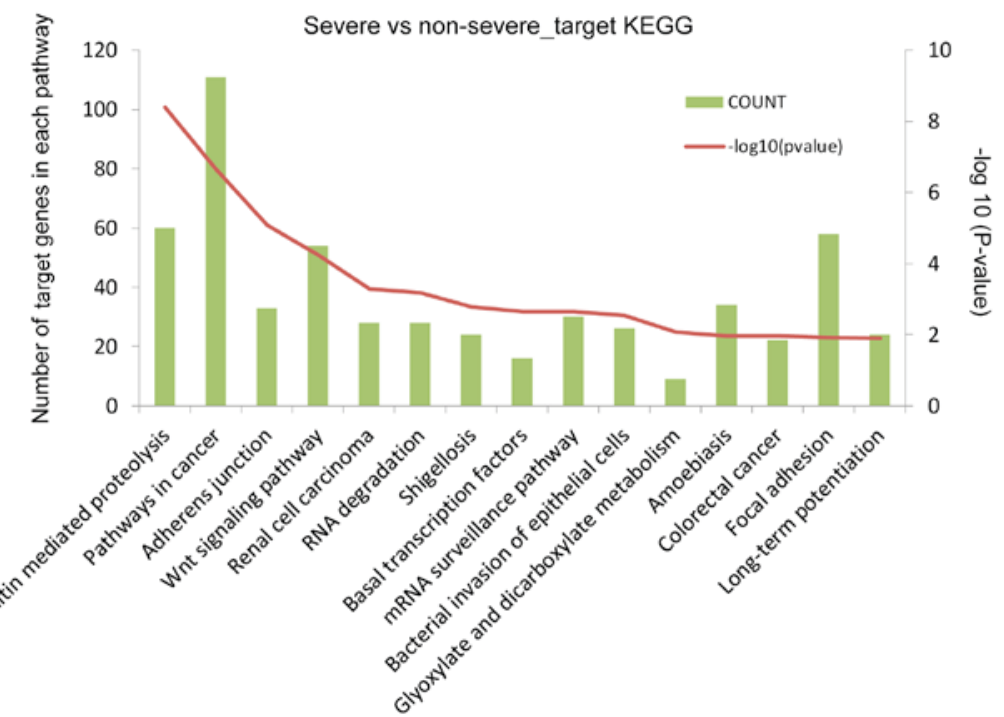

Figure 4. Enrichment analysis of key DE-miR target genes from comparisons of (A) non-severe pneumonia and control groups, and (B) severe and non-severe pneumonia groups. DE-miR, differentially expressed microRNA. -log10(P-value) represents the significance of the enriched pathway and was used to quantify and present the results more clearly. P-values were calculated following pathway enrichment and $\mathrm{P}<0.05$ was identified as the threshold for significant pathway selection.

aforementioned in the methods section. Five predominant protein interactors were identified: Ras homolog family member A (RhoA; degree, 28), $\beta$-catenin (CTNNB1; degree, 28), polymerase II subunit K (POLR2K; degree, 20), amyloid precursor protein (APP; degree, 20) and Kalirin, RhoGEF Kinase (KALRN; degree, 15). RHOA, CTNNB1, $P O L R 2 K$ and $A P P$ were predicted targets of hsa-let-7f-1.

\section{Discussion}

In the present study, 11 key DE-miRs were identified in pneumonia, including 6 upregulated miRNAs (including hsa-miR-200b and hsa-miR-455) and 5 downregulated miRNAs (including hsa-let-7f-1). Notably, these 11 miRNAs kept their upregulation/downregulation expression pattern in the three types of samples, which may reflect the three phases of pneumonia development. Enrichment analysis indicated that the target genes of DE-miRs identified from comparisons of non-severe pneumonia and the control, and severe pneumonia and non-severe pneumonia were highly associated with functions in the 'adherens junction' and 'Wnt signaling pathway'. In addition, KALRN, RhoA, CTNNB1, POLR2K and $A P P$ were determined to be crucial nodes in the PPI network of targets of the DE-miRs. KALRN was predicted as a target of hsa-miR-200b, while RHOA, CTNNB1, POLR2K and APP were predicted targets of hsa-let-7f-1.

miRNAs in the let-7 family function as regulators of cell proliferation. let-7 acts as an inhibitor during the development of lung cancer, indicating that its downregulation facilitates cell proliferation (27). In mice, let-7b and let-7c have been identified as important miRNAs in the lung restoration at the late stage of influenza pneumonia (27). In human non-small 


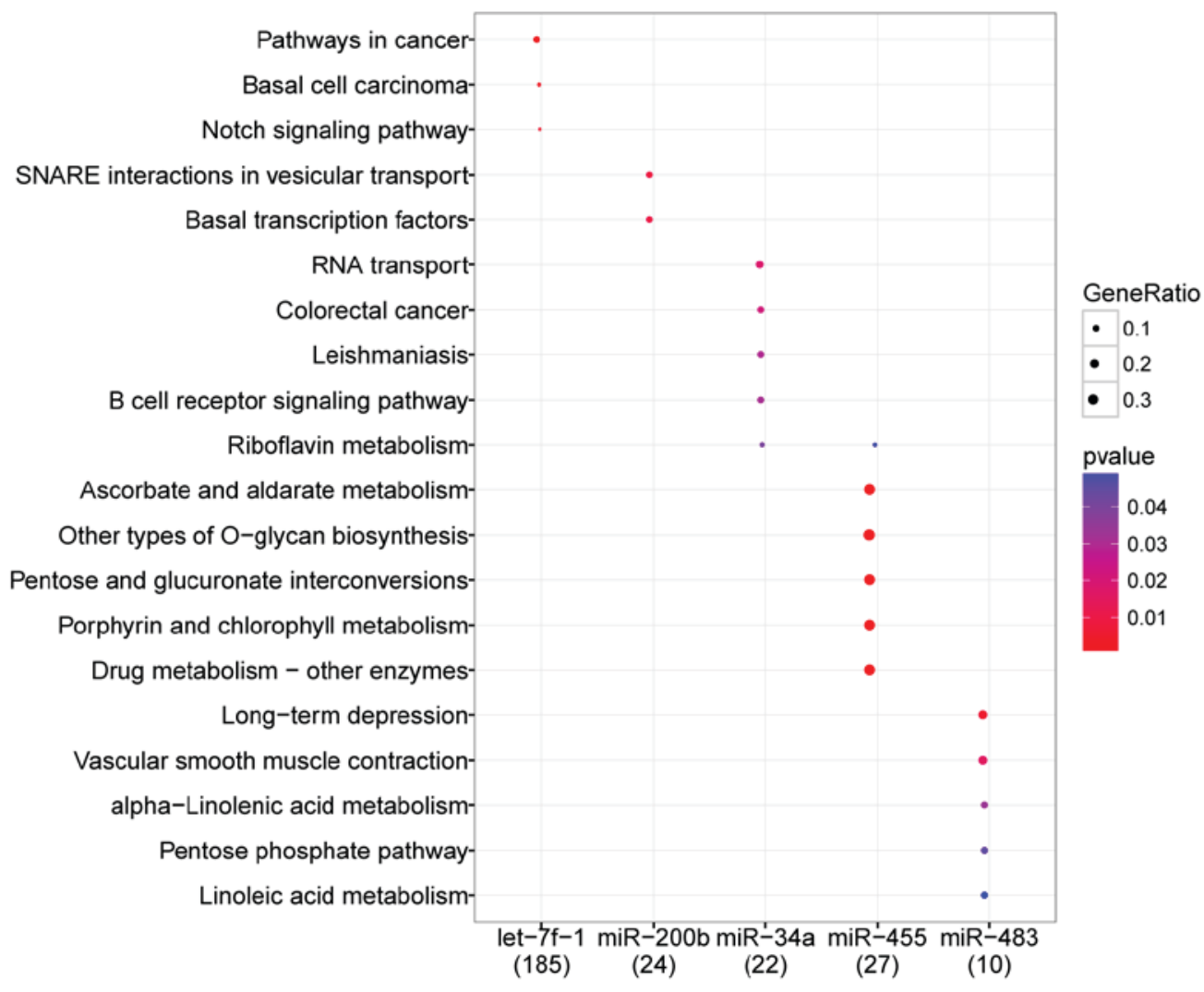

Figure 5. Enrichment analysis of the key DE-miRs. Names on the left are the pathway categories of the target genes. The circles of different sizes represent GeneRio, the ratio of the 'number of target genes enriched in a specific pathway' to the 'number of total genes in the pathway'. Different colors represent the P-values of the significance of different pathways, compared with the reference species. The numbers in brackets underneath the miRNA names indicate the numbers of identified genes in each pathway category. DE-miRs, differentially expressed microRNAs.

cell lung cancer (NSCLC), hsa-let-7c inhibits tumor metastasis and invasion via targeting the transcripts encoding integrin 3 and mitogen-activated protein kinase 3 (28). Previous studies have demonstrated that let- 7 family members are involved in many carcinogenic pathways, including the Notch signaling pathway, which is associated with a number of diseases in humans $(29,30)$. In the present study, hsa-let-7f-1 was found to be downregulated in pneumonia. Compared with the reference pathways in the reference species, this miRNA was significantly enriched in 'pathways in cancer' and the 'Notch signaling pathway', suggesting that let-7f-1 could function in these signaling pathways to regulate the progression of pneumonia. Downregulation of hsa-let-7f-1 may facilitate the invasion and metastasis of pneumonia-associated pathogens into lung epithelial cells, or reduce lung damage repair functions.

RhoA belongs to the Rho family, whose members are small guanosine-5'-triphosphate phosphatases and serve as molecular switches in signal transduction cascades. Overexpression of RhoA is associated with tumor cell proliferation and metastasis $(31,32)$. In antecedent influenza, Staphylococcus aureus strains are typically the cause of invasive infections, including pneumonia (33). A conserved surface protein of S. aureus, SpA (protein A), is overexpressed in the early stages of bacterial growth. Previous experiments in models of pneumonia have indicated that $\mathrm{SpA}$ activates the RhoA/Rho-associated protein kinase/myosin light chain protein signaling cascade, which results in contraction of the epithelial cytoskeleton that facilitates translocation via the paracellular junctions of the mucosal epithelium (34). Pseudomonas aeruginosa induces severe pneumonia and its type III secretion system facilitates the spread of this bacteria (35). A previous study in bovine pulmonary artery endothelial cells demonstrated that ExoS and ExoT, type III cytotoxins of $P$. aeruginosa, are responsible for increased vascular permeability in the lungs, through the activation of the RhoA signaling pathway (35). In the present study, RhoA was predicted as a target of hsa-let-7f-1, which was downregulated in pneumonia samples. These results suggest that the downregulation of hsa-let-7f-1 may induce the upregulation of $R H O$, activating the RhoA signaling pathway, thus increasing vascular permeability of the lungs and leading to pneumonia.

POLR2K encodes the smallest subunit of RNA polymerases II, which is the common subunit of three RNA polymerases (36). A previous study speculated that upregulation of POLR2K may facilitate the assembly of polymerase III, thus contributing to cell proliferation and cancer development (36). APP encodes a transmembrane precursor protein that is cleaved into peptides by secretases. In NSCLC, alternative splicing events influence the expression of AAP (37). In other types of cancer, increased expression of $A P P$ is associated with cell proliferation and tumorigenesis $(38,39)$. CTNNBI encodes a protein that is a key downstream component of the Wnt signaling pathway (40). CTNNB1 is associated with 


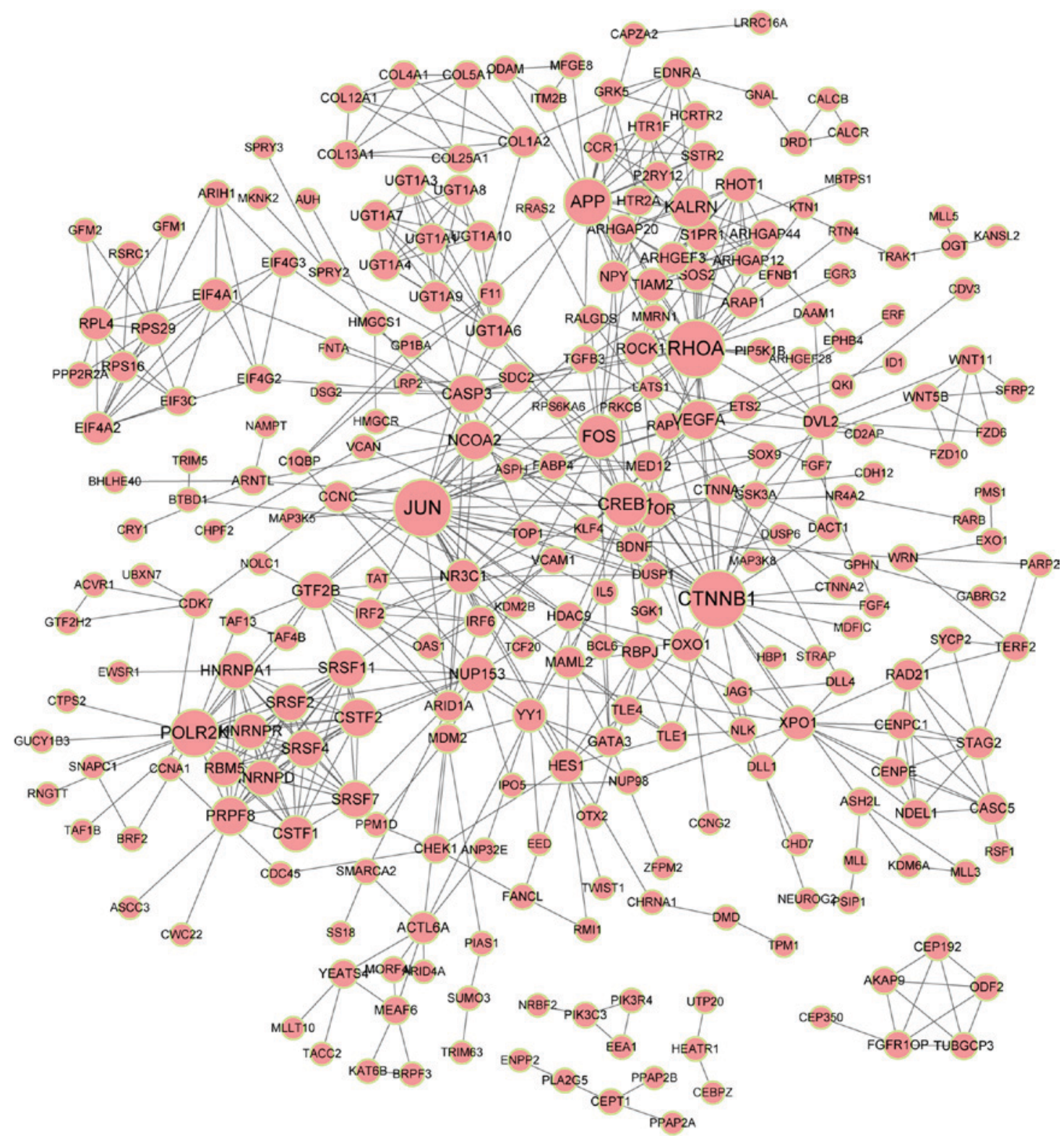

Figure 6. PPI network analysis of targets of the DE-miRs. Lines indicate interactions and the size of the circle indicates the number of interactions. PPI, protein-protein interaction; DE-miRs, differentially expressed microRNAs.

cadherin-mediated cell-cell adhesion systems and has been suggested as a biomarker for NSCLC prognosis (41). However, to the best of our knowledge, no studies investigating the association between POLR2K/CTNNBI/APP and pneumonia have been performed. In the present study, POLR $2 K, C T N N B 1$ and $A P P$ were downregulated in pneumonia and identified as targets of hsa-let-7f-1. In addition, their resulting proteins were functionally enriched in adherens junction and Wnt signaling pathways. These results suggest that POLR $2 K, C T N N B 1$ and $A P P$, regulated by hsa-let-7f-1, regulate pneumonia development through the adherens junction and Wnt signaling pathways.

miR-200b has an important role in lung cancer development; reportedly, aberrant expression of miR-200b in sputum may be used as a diagnostic marker for lung adenocarcinoma and squamouscell carcinoma (42). In a murine influenza pneumonia model, miR-200b-5p was predicted to be an important miRNA, connecting regulation of gene functions in repair (43). This suggests that the dysregulation of miR-200b may be a key mediator in pulmonary injury. KALRN encodes the protein kalirin, and decreased kalirin is implicated in the severity of allergic asthma (44). However, to the best of our knowledge, no current study elucidates the relationship between $K A L R N$ and pneumonia. In the present study, miR-200b was upregulated and predicted to target $K A L R N$, suggesting that expression of $K A L R N$ may be downregulated by miR-200b, and this downregulation may subsequently promote pneumonia development. A previous study reported that miR-455 suppresses NSCLC by targeting the transcript encoding Zinc finger E-box-binding homeobox 1 (45). This indicates that upregulation of miR-455 may inhibit the development of pneumonia. However, the specific mechanisms underlying this process need to be further investigated.

In conclusion, the present study identified a number of key DE-miRs, such as hsa-miR-200b, hsa-miR-455 and hsa-let-7f-1 in pneumonia. The results of bioinformatics analysis suggest that hsa-let-7f-1 contributes to the development of pneumonia 
by affecting cancer and Notch signaling pathways, through targeting and regulating transcripts encoding RhoA, CTNNB1, POLR2K and APP, while miR-200b may promote pneumonia via targeting $K A L R N$. In addition, the results of the present study indicate that hsa-miR-455 may serve as an inhibitor of pneumonia development. Further studies are required to validate these predictive results.

\section{Acknowledgements}

The present study was supported by grants from Welfare Industry Research Program of Ministry of Health (no. 201302017, 201502019), the National Natural Science Fund (No. 81272060, 81371561), the Hai Nan Natural Science Fund (20158315), the Youth Training Program of the PLA (no. 13QNP171), Beijing Scientific and Technologic Supernova Supportive Project (Z15111000030000/XXJH2015B100), PLA General Hospital Science and Technology Innovation Nursery Fund Project (16KMM56) and PLA Logistic Major Science and Technology Project (14CXZ005, AWS15J004, BWS14J041).

\section{References}

1. Organization WH: The top 10 causes of death. July 2013 Available at: who int/mediacentre/factsheets/fs310/en. Accessed July, 2014.

2. Wunderink RG and Waterer GW: Clinical practice. Community-acquired pneumonia. N Engl J Med 370: 543-551, 2014.

3. Said MA, Johnson HL, Nonyane BA, Deloria-Knoll M, O'Brien KL; AGEDD Adult Pneumococcal Burden Study Team, Andreo F, Beovic B, Blanco S, Boersma WG, et al: Estimating the burden of pneumococcal pneumonia among adults: A systematic review and meta-analysis of diagnostic techniques. PLoS One 8: e60273, 2013.

4. Rakha MA, Abdelmoneim AN, Farhoud S, Pièche S, Cousens S, Daelmans B and Bahl R: Does implementation of the IMCI strategy have an impact on child mortality? A retrospective analysis of routine data from Egypt. BMJ Open 3: pii.e001852, 2013.

5. Floyd J, Wu L, Hay Burgess D, Izadnegahdar R, Mukanga D and Ghani AC: Evaluating the impact of pulse oximetry on childhood pneumonia mortality in resource-poor settings. Nature 528: S53-S59, 2015.

6. Choi SH, Hong SB, Ko GB, Lee Y, Park HJ, Park SY, Moon SM, Cho OH, Park KH, Chong YP, et al: Viral infection in patients with severe pneumonia requiring intensive care unit admission. Am J Respir Crit Care Med 186: 325-332, 2012.

7. Berkley JA, Munywoki P, Ngama M, Kazungu S, Abwao J, Bett A, Lassauniére R, Kresfelder T, Cane PA, Venter M, et al: Viral etiology of severe pneumonia among Kenyan infants and children. JAMA 303: 2051-2057, 2010.

8. Sapru A, Hansen H, Ajayi T, Brown R, Garcia O, Zhuo H, Wiemels J, Matthay MA and Wiener-Kronish J: 4G/5G polymorphism of plasminogen activator inhibitor-1 gene is associated with mortality in intensive care unit patients with severe pneumonia. Anesthesiology 110: 1086-1091, 2009.

9. España PP, Capelastegui A, Bilbao A, Diez R, Izquierdo F, Lopez de Goicoetxea MJ, Gamazo J, Medel F, Salgado J, Gorostiaga I, et al: Utility of two biomarkers for directing care among patients with non-severe community-acquired pneumonia. Eur J Clin Microbiol Infect Dis 31: 3397-3405, 2012.

10. Wong KY, Huang X and Chim CS: DNA methylation of microRNA genes in multiple myeloma. Carcinogenesis 33: 1629-1638, 2012.

11. Abd-El-Fattah AA, Sadik NA, Shaker OG and Aboulftouh ML: Differential microRNAs expression in serum of patients with lung cancer, pulmonary tuberculosis, and pneumonia. Cell Biochem Biophys 67: 875-884, 2013.
12. Ramirez G, Uribe-Boll J, Cruz A, Jimenez L, Banales J, Romero S, Hidalgo A, Bautista E, Merino E and Moran J: Circulating microRNA profiles in patients with severe pneumonia associated to the A/H1N1 virus. Am J Respir Crit Care Med 189: A2694, 2014.

13. Ren S, Peng Z, Mao JH, Yu Y, Yin C, Gao X, Cui Z, Zhang J, Yi K, $\mathrm{Xu}$ W, et al: RNA-seq analysis of prostate cancer in the Chinese population identifies recurrent gene fusions, cancer-associated long noncoding RNAs and aberrant alternative splicings. Cell Res 22: 806-821, 2012.

14. Wang Z, Gerstein M and Snyder M: RNA-Seq: A revolutionary tool for transcriptomics. Nat Rev Genet 10: 57-63, 2009.

15. Weiwu D: Guidelines for the diagnosis and treatment of community acquired pneumonia. Chinese J Tuberculosis Respiratory Dis 29: 651-655, 2001.

16. Griffiths-Jones S, Saini HK, van Dongen S and Enright AJ: miRBase: Tools for microRNA genomics. Nucleic Acids Res 36: D154-D158, 2008.

17. Smyth GK: Limma: Linear models for microarray data. In: Bioinformatics and Computational Biology Solutions Using R and Bioconductor. Springer, New York, NY, 397-420, 2005.

18. Benjamini Y and Hochberg Y: Controlling the false discovery rate: A practical and powerful approach to multiple testing. J Royal Statistical Society Series B (Methodological) 57: 289-300, 1995.

19. John B, Enright AJ, Aravin A, Tuschl T, Sander C and Marks DS: Human microRNA targets. PLoS Biol 2: e363, 2004.

20. Palmieri D, Capponi S, Geroldi A, Mura M, Mandich P and Palombo D: TNFa induces the expression of genes associated with endothelial dysfunction through p38MAPK-mediated down-regulation of miR-149. Biochem Biophys Res Commun 443: 246-251, 2014.

21. Harris MA, Clark J, Ireland A, Lomax J, Ashburner M, Foulger R, Eilbeck K, Lewis S, Marshall B, Mungall C, et al: The gene ontology (GO) database and informatics resource. Nucleic Acids Res 32 (Database Issue): D258-D261, 2004.

22. Kanehisa M, Goto S, Sato Y, Furumichi M and Tanabe M: KEGG for integration and interpretation of large-scale molecular data sets. Nucleic Acids Rese 40 (Database Issue): D109-D114, 2012.

23. Dennis G Jr, Sherman BT, Hosack DA, Yang J, Gao W, Lane HC and Lempicki RA: DAVID: Database for annotation, visualization, and integrated discovery. Genome Biol 4: P3, 2003.

24. Yu G, Wang LG, Han Y and He QY: ClusterProfiler: An R package for comparing biological themes among gene clusters. OMICS 16: 284-287, 2012.

25. Szklarczyk D, Franceschini A, Wyder S, Forslund K, Heller D, Huerta-Cepas J, Simonovic M, Roth A, Santos A, Tsafou KP, et al: STRING v10: Protein-protein interaction networks, integrated over the tree of life. Nucleic Acids Res 43: D447-D452, 2015.

26. Kohl M, Wiese S and Warscheid B: Cytoscape: Software for visualization and analysis of biological networks. Methods Mol Biol 696: 291-303, 2011.

27. Tan KS, Choi H, Jiang X, Yin L, Seet JE, Patzel V, Engelward BP and Chow VT: Micro-RNAs in regenerating lungs: An integrative systems biology analysis of murine influenza pneumonia. BMC Genomics 15: 587, 2014.

28. Zhao B, Han H, Chen J, Zhang Z, Li S, Fang F, Zheng Q, Ma Y, Zhang J, Wu N and Yang Y: MicroRNA let-7c inhibits migration and invasion of human non-small cell lung cancer by targeting ITGB3 and MAP4K3. Cancer Lett 342: 43-51, 2014.

29. Wang X, Cao L, Wang Y, Wang X, Liu N and You Y: Regulation of let-7 and its target oncogenes (Review). Oncol Lett 3: 955-960, 2012.

30. Liu XS, Chopp M, Zhang RL, Tao T, Wang XL, Kassis H, Hozeska-Solgot A, Zhang L, Chen C and Zhang ZG: MicroRNA profiling in subventricular zone after stroke: MiR-124a regulates proliferation of neural progenitor cells through Notch signaling pathway. PLoS One 6: e23461, 2011.

31. Pillé JY, Denoyelle C, Varet J, Bertrand JR, Soria J, Opolon P, Lu H, Pritchard LL, Vannier JP, Malvy C, et al: Anti-RhoA and Anti-RhoC siRNAs inhibit the proliferation and invasiveness of MDA-MB-231 breast cancer cells in vitro and in vivo. Mol Ther 11: 267-274, 2005.

32. Chan CH, Lee SW, Li CF, Wang J, Yang WL, Wu CY, Wu J, Nakayama KI, Kang HY, Huang HY, et al: Deciphering the transcriptional complex critical for RhoA gene expression and cancer metastasis. Nat Cell Biol 12: 457-467, 2010.

33. DeLeo FR and Musser JM: Axis of coinfection evil. J Infect Dis 201: 488-490, 2010 
34. Soong G, Martin FJ, Chun J, Cohen TS, Ahn DS and Prince A: Staphylococcus aureus protein A mediates invasion across airway epithelial cells through activation of RhoA GTPase signaling and proteolytic activity. J Biol Chem 286: 35891-35898, 2011.

35. Ganter MT, Roux J, Su G, Lynch SV, Deutschman CS Weiss YG, Christiaans SC, Myazawa B, Kipnis E and Wiener-Kronish JP: Role of small GTPases and alphavbeta5 integrin in Pseudomonas aeruginosa-induced increase in lung endothelial permeability. Am J Respir Cell Mol Biol 40: 108-118, 2009.

36. Lin Y, Li Z, Ozsolak F, Kim SW, Arango-Argoty G, Liu TT, Tenenbaum SA, Bailey T, Monaghan AP, Milos PM and John B: An in-depth map of polyadenylation sites in cancer. Nucleic Acids Res 40: 8460-8471, 2012.

37. Misquitta-Ali CM, Cheng E, O'Hanlon D, Liu N, McGlade CJ, Tsao MS and Blencowe BJ: Global profiling and molecular characterization of alternative splicing events misregulated in lung cancer. Mol Cell Biol 31: 138-150, 2011.

38. Krause K, Karger S, Sheu SY, Aigner T, Kursawe R, Gimm O, Schmid KW, Dralle H and Fuhrer D: Evidence for a role of the amyloid precursor protein in thyroid carcinogenesis. J Endocrinol 198: 291-299, 2008.
39. Takayama K, Tsutsumi S, Suzuki T, Horie-Inoue K, Ikeda K, Kaneshiro K, Fujimura T, Kumagai J, Urano T, Sakaki Y, et al: Amyloid precursor protein is a primary androgen target gene that promotes prostate cancer growth. Cancer Res 69: 137-142, 2009.

40. Polakis P: Casein kinase 1: A Wnt'er of disconnect. Curr Biol 12: R499-R501, 2002.

41. Woenckhaus M, Merk J, Stoehr R, Schaeper F, Gaumann A, Wiebe K, Hartmann A, Hofstaedter F and Dietmaier W: Prognostic value of FHIT, CTNNB1, and MUC1 expression in non-small cell lung cancer. Hum Pathol 39: 126-136, 2008.

42. Shen J, Liao J, Guarnera MA, Fang H, Cai L, Stass SA and Jiang F: Analysis of MicroRNAs in sputum to improve computed tomography for lung cancer diagnosis. J Thorac 9: 33-40, 2014.

43. Tan KS, Choi H, Jiang X, Yin L, Ju ES, Patzel V, Engelward BP and Chow VT: Micro-RNAs in regenerating lungs: An integrative systems biology analysis of murine influenza pneumonia. BMC Genomics 15: 1-13, 2014.

44. Dewan AT, Egan KB, Hellenbrand K, Sorrentino K, Pizzoferrato N, Walsh KM and Bracken MB: Whole-exome sequencing of a pedigree segregating asthma. BMC Medical Genetics 13: 1-9, 2012.

45. Li YJ, Ping C, Tang J and Zhang W: MicroRNA-455 suppresses non-small cell lung cancer through targeting ZEB1. Cell Biol Int 40: 621-628, 2016. 